\title{
Thrombektomie lohnt sich
}

Fragestellung: Ist die endovaskuläre Therapie bei intrakraniellen Verschlüssen groBer Arterien im vorderen Kreislauf einer systemischen Thrombolyse überlegen?

Hintergrund: Vor zwei Jahren wurden die Ergebnisse von drei randomisierten Studien vorgestellt, in denen eine Thrombektomie mit einer systemischen Thrombolyse oder anderen Therapien verglichen wurde. Diese Studien waren negativ. In den letzten zehn Monaten wurden fünf weitere Studien publiziert, in denen die Thrombektomie zusätzlich zu einer systemischen Thrombolyse gegen eine reine Thrombolyse verglichen wurde. Diese Studien waren positiv. Die vorliegende Publikation ist die erste Metaanalyse aller Studien und getrennt davon der letzten fünf Studien.

Patienten und Methodik: In die Metaanalyse gingen die drei alten Studien aus 2013 ein (IMS III, MR Rescue und SYNTHESIS) sowie aus 2015 die Studien MR CLEAN, ESCAPE, EXTEND-IA, SWIFT PRIME und REVASCAT. Der primäre Endpunkt der Metaanalyse war ein gutes funktionelles Ergebnis definiert als ein Wert auf der modifizierten Rankin Skala zwischen 0 und 2 nach 90 Tagen. Andere Endpunkte waren die Sterblichkeit und symptomatische intrazerebrale Blutungen.

Ergebnisse: Die primäre Metaanalyse schloss acht randomisierte Studien mit 2.423 Patienten ein, die überwiegend Verschlüsse der distalen Arteria carotis interna oder der proximalen Arteria cerebri media aufwiesen. Über alle Studien hinweg erhöhte die endovaskuläre Therapie die Wahrscheinlichkeit eines guten funktionellen Outcome nach 90 Tagen mit einer Odds Ratio (OR) von 1,73 (Number needed to treat [NNT] 9,3). Die Sterblichkeit war mit einer OR von 0,89 reduziert, aber statistisch nicht signifikant. Die Rate symptomatischer intrakranieller Blutungen war mit einer OR von 1,07 nicht unterschiedlich. Eine Analyse der fünf Studien aus dem Jahr 2015 zeigt eine OR von 2,42 für den primären Endpunkt einer funktionellen Unabhängigkeit mit einer NNT von 5, eine OR von 0,80 (nicht signifikant) für die Sterblichkeit und eine OR von 1,08 für sympto-

Sardar P, Chatterjee S, Giri J et al. Endovascular therapy for acute ischaemic stroke: a systematic review and meta-analysis of randomized trials. Eur Heart J 2015; Epub ahead of print matische intrazerebrale Blutungen.

Schlussfolgerungen: Eine Metaanalyse älterer und neuerer Studien zur Thrombektomie zeigt eine signifikante Überlegenheit dieses Vorgehens für einen guten funktionellen Outcome.

\section{- Kommentar von Hans-Christoph Diener, Essen}

\section{Geeignete Patienten rasch identifizieren}

Diese erste Metaanalyse belegt, was die einzelnen Studien bereits gezeigt hatten, nämlich eine Überlegenheit der frühen endovaskulären Therapie in Kombination mit einer systemischen Thrombolyse gegenüber einer alleinigen systemischen Thrombolyse. Rein methodisch mag es richtig sein, die drei alten negativen Studien mit den neuen Studien zusammen auszuwerten. Inhaltlich macht es wenig Sinn, da die drei alten Studien schwerwiegende methodische Probleme hatten. Nimmt man die fünf neuen Studien zum Maßstab, ist eine Number needed to treat von 5 für einen guten Outcome nach 90 Tagen spektakulär.

Ziel muss jetzt sein, die Patienten zu identifizieren, die sich optimal für diese neue Technik eignen, und die Infrastruktur der Patientenversorgung dahingehend zu optimieren, dass Patienten, die für diese Therapie infrage kommen, auch möglichst schnell in entsprechende Schlaganfallzentren überwiesen werden. 